\title{
PERCEPÇÃO DE DOR E ALTERAÇÕES MUSCULOESQUELÉTICAS EM ATLETAS DE BASQUETE NA PREPARAÇÃO PARA OS JOGOS UNIVERSITÁRIOS DE 2014
}

\author{
Mariana Moreira*, Marília Ferreira dos Santos*, Gustavo Sperandio Barros**, Tharsila dos Santos \\ Nascimento**, Anderson Delano Araújo****, Mansueto Gomes Neto***** \\ Autor para correspondência: Mariana Moreira - mmarianamoreira@gmail.com \\ * Departamento de Fisioterapia, Curso de Fisioterapia da Universidade Federal da Bahia - UFBA, Salvador-BA, Brasil \\ ** Departamento médico da Confederação Brasileira do Desporto Universitário, Salvador-BA, Brasil \\ *** Curso de Fisioterapia da Universidade Tiradentes de Sergipe- UNIT, Aracaju-SE,Brasil \\ **** Docente da Faculdade São Salvador, Salvador - BA, Brasil \\ ***** Departamento de Fisioterapia, Curso de Fisioterapia da Universidade Federal da Bahia - UFBA, Docente do programa \\ de Pós Graduação em Medicina e Saúde - UFBA Salvador - BA, Brasil.
}

\section{Resumo}

Objetivo: Avaliar a percepção de dor e alterações musculoesqueléticas em jogadores de basquete na preparação para o $62^{\circ}$ jogos Universitários Brasileiros. Métodos: Foi realizado um estudo analítico de caráter retrospectivo com atletas de basquete, de ambos os sexos registrados no $62^{\circ}$ jogos Universitários Brasileiros de 2014. Os atletas foram avaliados antes do início dos jogos, os atletas responderam perguntas sobre a percepção de dor musculoesquelética e ocorrência de alguma lesão durante a preparação para os jogos universitários. Resultados: Foi realizada estatística descritiva e inferencial utilizando o software SPSS versão 20.0.Foram entrevistados 296 jogadores de basquete distribuídos em 34 equipes, sendo 19 masculinas e 15 femininas. A idade média foi de 22,08 \pm 3,62 anos e 170 $(57,4 \%)$ foram do sexo masculino. Dos 296 atletas, 185 (62,5\%) relataram sentir alguma dor e 213 (72\%) relataram a presença de alguma lesão durante a preparação para a competição. 73 (39,5\%) relataram sentir dor no joelho, seguido da coluna 45 (24,3\%), 24(13\%) membro superior, 18 (9,7\%) tornozelo e pé. Quando comparada a amostra em relação ao sexo não houve diferença estatisticamente significativa em relação à idade, percepção de dor e regiões corporais afetadas. Já em relação à ocorrência de lesões na preparação, foi observada uma diferença estatisticamente significativa, com maior prevalência nas equipes do sexo masculino. Conclusão: Existe uma alta prevalência de dor e alterações musculoesqueléticas em atletas de basquete durante preparação para competição. Estudos que avaliem fatores associados a dor são necessários.

Palavras-chave: Dor; Basquetebol; Lesão. 


\title{
PERCEPTION OF PAIN AND MUSCULOSKELETAL DISORDERS IN BASKETBALL ATHLETES IN THE PREPARATION FOR UNIVERSITY GAMES 2014
}

\begin{abstract}
Objective: To assess the perception of pain and musculoskeletal disorders in basketball players on preparation for the 62nd Brazilian University games. Methods: We conducted a retrospective analytical study with basketball athletes of both sexes registered in 62nd Brazilian University Games 2014. The athletes were evaluated before the Games began, answering questions about the perception of musculoskeletal pain and occurrence of an injury while preparing for college games. It was employed a descriptive and inferential statistics analysis with the SPSS software version 20.0. Results: We interviewed 296 basketball players divided into 34 teams, 19 were male and 15 were female. The average age was $22.08 \pm 3.62$ years and 170 (57.4\%) were male. Of the 296 athletes, $185(62.5 \%)$ reported feeling some pain and $213(72 \%)$ reported the presence of an injury while preparing for the competition. 73 (39.5\%) reported pain in the knee, followed by pain in the column 45 $(24.3 \%), 24$ (13\%) in the upper limb, 18 (9.7\%) in the ankle and foot. When the sample was assessed taking in consideration the genders, there was no statistically significant difference in relation to age, pain perception and affected body regions. In relation to the occurrence of injuries in the preparation, a statistically significant difference was observed with higher prevalence in male teams. Conclusion: There is a high prevalence of musculoskeletal pain and changes in basketball athletes during preparation for competition. Further studies to evaluate factors associated with pain are needed.
\end{abstract}

Keywords: Pain; Basketball; Injury.

\section{INTRODUÇÃO}

O basquete é um esporte de grande movimentação e coordenação, apresenta os movimentos básicos dos esportes, como aceleração, desaceleração, mudança de direção, pivoteios e possui como características principais esforços breves e intensos, realizados em diversos ritmos, com saltos, corridas e movimentos coordenados, ${ }^{(1,2)}$ o que o torna um esporte potencialmente lesivo. Dentre os vários fatores que geram desgastes na estrutura física dos atletas, os frequentes impactos advindos das quedas que, (3) não são exclusivas de esportes de combate, são os grandes vilões dos atletas cujas modalidades possuem fundamentos impactantes. Devido à exigência do esporte, treinamento inten- so, contato e impactos, o esporte apresenta alta frequência de lesões durante a preparação e nas competições. $(4,5)$

Apesar do uso constante dos membros superiores no basquete, o número de lesões em membros superiores é menor que lesões dos membros inferiores. O tipo de lesão mais encontrada foi a lesão articular, representando 58,97\% do total de lesões. ${ }^{(2)}$ A entorse de tornozelo foi o tipo de lesão articular mais relatado na equipe masculina, seguida por tendinopatia, distensão muscular e fraturas. Porém, na equipe feminina a entorse de joelho foi a lesão articular mais frequente. 
O esporte apresenta uma alta incidência de lesões. Estudos relacionados ao basquete ainda são escassos. Estudos que identifiquem a presença de dor e/ou lesões musculoesqueléticas durante a preparação para campeonatos ou competições podem ajudar a equipe de saúde e de técnicos a nortearem seus trabalhos, no sentido de um treinamento mais funcional e no aprimoramento dos recursos a serem usados para o tratamento das lesões.

A combinação entre o treinamento específico e a abordagem científica pode contribuir para o desenvolvimento de métodos de treinamento que produzam atletas mais adaptados às demandas competitivas e, assim, contribuir para melhorar o desempenho das equipes de basquetebol. (6) Sendo assim, o objetivo do estudo foi avaliar a percepção de dor e alterações musculoesqueléticas em jogadores de basquete na preparação para o $62^{\circ}$ jogos Universitários Brasileiros.

\section{METODOLOGIA}

Foi realizado um estudo analítico de caráter retrospectivo, quantitativo. Foram incluídos atletas de basquete com idade maior de 18 anos de ambos os sexos registrados no $62^{\circ}$ jogos Universitários Brasileiros que ocorreu entre 29 de outubro e o9 de novembro de 2014, em Aracajú, Sergipe, Brasil. Os atletas foram convidados a participar, os que aceitaram assinavam o Termo de Consentimento Livre e Esclarecido (TCLE). Estudo foi aprovado no Comitê de Ética da Faculdade de Medicina da Bahia com parecer: 833.228

Inicialmente foram coletados os dados sócios demográficos através de questionários previamente elaborados pelos autores. Uma das perguntas incluídas na entrevista foi sobre a saúde do atleta, na qual tinha como opções: excelente, boa, regular e ruim.

Antes do início das partidas foi perguntado aos atletas sobre a sua percepção de dor musculoesquelética e ocorrência de alguma lesão durante a preparação para os jogos universitários. Os dados foram anotados e classificados segundo critérios preestabelecidos para localização, etiologia e diagnóstico se estabelecido. Após a competição os dados foram tabulados e analisados.

Os dados de variáveis contínuas foram analisados com medidas de tendência central e dispersão e expressos como médias e desvio-padrão. Os dados de variáveis dicotômicas ou categóricas foram analisados com medidas de frequência e expressos como porcentagens. Como os dados foram normalmente distribuídos, analisados pelo teste Shapiro-Wilk, o teste $\mathrm{t}$ de student para amostras independentes foi utilizado para comparar as diferenças das médias das variáveis continuas e o teste Qui-Quadrado para as variáveis qualitativas entre equipes masculinas e femininas. $O$ nivel de significância estabelecido foi de 5\%. A análise estatística foi realizada com o uso do software SPSS (StatisticalPackage for the Social Sciences) for Windows (versão 20.0).

\section{RESULTADOS}

Foram convidados a participar do estudo 332 atletas de ambos os sexos antes do início dos jogos. Destes, 27 não aceitaram participar do estudo e nove não preencheram os formulários com dados de identificação e foram excluídos do estudo. A amostra foi composta de 296 jogadores de basquete distribuídos em 34 equipes, sendo 19 masculinas e 15 femininas. A idade média foi de 22,08 $\pm 3,62$ anos, e $170(57,4 \%)$ foram do sexo masculino, sendo 281 $(94,9 \%)$ solteiros e $15(5,1 \%)$ casados. Quando perguntados pela sua percepção de saúde 147 (49,7\%) responderam excelente, 135 (45,6\%) como boa, 12 $(4,1 \%)$ responderam regular e apenas $2(0,7 \%)$ consideraram a saúde como ruim.

Em relação à percepção de dor, dos 296 atletas $185(62,5 \%)$ relataram sentir alguma dor durante a preparação para a competição. Destes 73 (39,5\%) relataram sentir dor no joelho, seguido da coluna 45 (24,3\%), 24(13\%) membro superior, 18 (9,7\%) tornozelo e pé e outras regiões como perna, coxa e 
cabeça que totalizaram $13,6 \%$. Em relação à ocorrência de alguma lesão musculoesquelética prévia, $213(72 \%)$ relataram a presença de lesão.

Quando comparada a amostra em relação ao sexo não houve diferença estatisticamente significativa em relação à idade média $(p=0,79)$, percepção de dor $(p=0,25)$ e em relação às regiões corporais afetadas $(p=0,77)$ entre os atletas das equipes masculinas e femininas. Já em relação à ocorrência de lesões prévias a competição, foi observada uma prevalência de $76,5 \%$ em atletas das equipes masculinas e $65,9 \%$ nas equipes femininas, tendo uma diferença estatisticamente significativa $p=0,031$. Os dados referentes às características sociodemograficas bem como da percepção e localização da dor estão descritas na tabela 1.

Tabela 1 - Dados sociodemográficos e da percepção de dor e alteração musculoesquelética por sexo

\begin{tabular}{lcc}
\hline & EquIPES MASCULINAS & EQUIPES FEMININAS \\
& $\mathrm{N}=170$ & $\mathbf{N}=126$ \\
\hline Idade (anos) & $22,12 \pm 3,50$ & $22,01 \pm 3,78$ \\
Frequência de Treino (x sem) & $1,93 \pm 0,52$ & $1,90 \pm 0,65$ \\
Saúde & $91(53,5 \%)$ & $53(44,4 \%)$ \\
Excelente & $74(43,5 \%)$ & $61(48,4 \%)$ \\
Boa & $5(2,9 \%)$ & $7(5,6 \%)$ \\
Regular & - & $2(1,6 \%)$ \\
Ruim & $103(60,6 \%)$ & $82(65,1 \%)$ \\
Percepção de Dor & & \\
Localização & $3(2,9 \%)$ & $1(1,2 \%)$ \\
Cabeça & $13(12,5 \%)$ & $11(13,6 \%)$ \\
Membro Superior & $25(24 \%)$ & $20(24,7 \%)$ \\
Coluna & $4(3,8 \%)$ & $5(6,2 \%)$ \\
Coxa & $40(38,5 \%)$ & $33(40,7 \%)$ \\
Joelho & $6(5,8 \%)$ & $6(7,4 \%)$ \\
Perna & $13(12,5)$ & $5(6,2 \%)$ \\
Tornozelo/pé & $130(76,5 \%)$ & $83(65,9 \%)^{*}$ \\
Lesão musculesquelética prévia & & \\
\hline
\end{tabular}

${ }^{*} p<0.05$

\section{DISCUSSÃO}

O objetivo deste estudo foi avaliar a percepção de dor e alterações musculoesqueléticas em jogadores de basquete. No presente estudo foi observada alta ocorrência de dor e presença de lesão musculoesquelética em atletas de basquete durante a preparação para os jogos universitários, corroborando outros estudos que avaliaram a presença de lesões durante a prática do basquete..$^{(6,8)}$
No basquete, movimentos de alto risco como saltos, aterrissagens, giros, pivoteios, corridas e quedas, que são comuns, podem aumentar o risco de dor e lesões musculoesqueléticas, seja durante treinamentos ou em jogos competitivos. ${ }^{(9)}$ Vários fatores que ocasionam desgaste na estrutura física de atletas, a forma de aterrissagem e o frequente impacto advindo de choques ou quedas, são fatores desencadeante de lesões osteomusculares. ${ }^{(10)}$

A alta frequência de dor percebida pelos atletas pode estar associada às características próprias da 
modalidade esportiva, mas também pela necessidade do atleta de se sentir mais preparado para a competição, e assim aceitar a dor como uma experiência necessária para alcançar melhor rendimento. $\mathrm{Na}$ tentativa de alcançar o melhor desempenho possível o atleta tende a atuar sobre a linha tênue que difere a dor originada pela fadiga e a dor originada por lesão(11).

Apesar da percepção de dor entre a maioria dos atletas, 95,3\% destes informaram que a saúde estava excelente ou boa, o que denota que mesmo na presença de dor a maior parte dos atletas não associou a dor à redução da saúde. Culturalmente no esporte de alto nível impõe-se que o atleta deva ser resistente à dor, levando, em algumas situações, a prática esportiva, mesmo na presença de dor e possível lesão. ${ }^{(12)} \mathrm{Em}$ um estudo realizado durante os Jogos Pan Americanos no Brasil em 2007, foi descrito que $22,1 \%$ da delegação de atletas brasileiros participaram da competição com algum tipo de lesão. ${ }^{(13)}$

Os dados do presente estudo demonstram alta taxa de ocorrência de lesões mesmo durante a preparação para a competição. Apesar deste resultado expressivo de $72 \%$ de lesões durante a preparação, as lesões durante as competição demonstram resultados semelhantes. Autores ${ }^{(4,5)}$ apresentaram dados de acompanhamento de atletas durante os treinos e jogos de basquete, mostrando taxas similares de lesões do sistema locomotor entre treinos e jogos. No presente estudo o fato dos atletas passarem mais tempo treinando que em competição pode explicar a alta frequência de lesões prévias.

Em relação à localização da lesão, observa-se que o local mais acometido foi o joelho, seguido pela coluna e membro superior, concordando com o estudo de Silva et al onde o joelho foi a região mais afetada. Estudos ${ }^{(13-16)}$ apontam os membros inferiores como tendo maior frequência de lesões, comparada aos membros superiores e tronco, no qual estes apresentam uma frequência similar. A prevalência de lesões durante a temporada de 2002 da seleção brasileira, foi de $48 \%$ para membros inferiores seguido de $13,7 \%$ para membros superior e frequência igual para tronco, dados semelhantes ao encontrado no presente estudo. ${ }^{(17)}$

Apesar da alta taxa de lesão em membros inferiores, chama atenção a frequência de lesões em coluna, $24,4 \%$ foram registradas no estudo. Este resultado pode estar associado a fatores relacionados às características próprias do esporte, como salto, aterrissagem e giros que promovem impacto sobre os membros inferiores e coluna vertebral ${ }^{(18,19)}$ bem como a fatores posturais que não foram avaliados no presente estudo.

A equipe masculina mostra-se mais propensa a sofrer lesões quando comparada a feminina(4). No presente estudo foi observada uma diferença significativa de 10,6\% em ocorrência de lesões nas equipes masculinas comparada às equipes femininas. Esta diferença entre sexos pode estar relacionada a diferenças inerentes e impostas durante jogos, como intensidade do jogo, velocidade alcançada, altura do salto e maior impacto entre homens comparado a mulheres. ${ }^{(20)}$

Este estudo apresenta algumas limitações. Por se tratar de estudo retrospectivo pode ter acontecido viés de memória por parte dos atletas. A recusa na participação e exclusões totalizou mais que $10 \%$ dos atletas, o que pode ter afetado o resultado encontrado. Ausência de avaliação de fatores biopsicossociais para verificar a relação com a presença de lesão e a existência da dor. Estudos prospectivos devem ser realizados com objetivo de acompanhar esses atletas durante toda a temporada obtendo informações durante a preparação, mas também durante as competições, com intuito de identificar fatores que possam estar associados à presença de dor e lesões durante a preparação e a competição. É necessário compreender melhor a relação entre a dor e presença de lesão e como estas podem afetar a saúde dos atletas a longo prazo. Os resultados permitem compreender que a avaliação, identificação das lesões e da dor, pode contribui para direcionar os programas de prevenção que devem ser implementados. 


\section{CONCLUSÃO}

O presente estudo encontrou uma alta percepção de dor e lesões musculoesqueléticas por parte dos atletas de basquete, sendo a ocorrência de lesões durante a preparação para os jogos universitários maior nas equipes masculinas que femininas.

\section{REFERÊNCIAS}

1. Acquesta FM, Peneireiro GM, Bianco R, Amadio AC, Serrão JC. Características dinâmicas de movimentos seleccionados do basquetebol. Rev Port Cien Desp. 2007;7(2):174-82.

2. Almeida AF, Tonin JP, Navega MT. Caracterização de lesões desportivas no basquetebol. Fisioter Mov. 2013;26(2):361-368.

3. Jeffrey B. Taylor, Kevin R. Ford, Anh-Dung Nguyen, Lauren N. Terry, Eric J. Hegedus Prevention of Lower Extremity Injuries in Basketball: A Systematic Review and MetaAnalysis Sports Health. 2015;7(5):392-398

4. Messina DF, Farney WC, Delee JC. The incidence of injury in Texas high school basketball.A prospective study among male and female athletes. Am J Sports Med 1999;27:294-9.

5. Gantus MC, Assumpção JD. Epidemiologia das lesões do sistema locomotor em atletas de basquetebol. Acta Fisiátr. 2002;9:77-84.

6. Dario, BES; Barquilha G, Marques RM. Lesões esportivas: um estudo com atletas do basquetebol bauruense. Rev Bras Ciênc Esporte.2010;31(3):256-260.

7. ALMAS Saulo Peters. Análise das estatísticas relacionadas ao jogo que discriminam as equipes vencedoras das perdedoras no basquetebol profissional brasileiro. Rev Bras Educ Fís. Esp. 2015;29(4):551-558.

8. . Darrow CJ, Collins CL, Yard EE, Comstock RD. Epidemiology of Severe Injuries Among United States High School Athletes: 2005-2007. Am J Sports Med. 2009;37(9):1798-1805.

9. Silva AS, Abdalla RJ, Fisberg M. Incidência de lesões musculoesqueléticas em atletas de elite do basquetebol feminino. Acta Ortop Bras. 2007;15(1):43-46
10. Roquete J. Sistematização e análise das técnicas de controle das quedas no judô (“ukemis). Revista Ludens.1994;14(2):45-53.

11. Moura PV, Silva EAP, Silva PPC, Freitas CMS, Caminha IO. O significado da dor física na prática do esporte de rendimento. Rev Bras Ciênc Esporte. 2013;35(4):1005-1019.

12. Wiese-Bjornstal DM. Psychology and socioculture affect injury risk, response, and recovery in high-intensity athletes: a consensus statement. Scand J Med Sci Sports. 2010;20:103-111.

13. Lopes AD. et al. Brazilian physiotherapy services in the 2007 Pan-American games: injuries, their anatomical location and hysiotherapeutic procedures. Phys Ther Sport. 2009;10:67-70.

14. McKay GD, Goldie PA, Payne WR, Oakes BW, Watson LF. A prospective study of injuries in basketball: a total profile and comparison by gender and standard of competition. J Sci Med Sport. 2001;4(2):196-211.

15. Burnham BR, Copley GB, Shim MJ, Kemp PA. Mechanism of basketball injuries reported to the HQ Air Force Safety Center: A 10-year descriptive study, 1993-2002. Am J Prev Med. 2010;38(S1):S134-40

16. Moreira P. Prevalência de lesões das equipes de base e adultas que representaram a Seleção Brasileira de Basquete em 2003. Rev Bras Ciênc Mov. 2006;14(2):71-8.

17. Calatayud J, Borreani S, Colado JC, Flandez J, Page P, Andersen LL. Exercise and ankle sprain injuries: A comprehensive review. Phys Sportsmed.2014;42:88-93.

18. Moreira P, Gentil D, Oliveira C. Prevalence of injuries of Brazilian Basketball National Team during $2 \mathrm{OO} 2$ season. Rev Bras Med Esporte 2003;9(5):258-262.

19. Adrian M, Cooper J. Biomechanics of selected team sports. The biomechanics of Human Movement. 1995;393-425.

2O. Waterman BR, Belmont PJ Jr, Cameron KL, Svoboda SJ, Alitz CJ, Owens BD. Risk factor for syndesmotic and medial ankle sprain: role of sex, sport, and level of competition. Am J Sports Med. 2011;992-998. 